\title{
Worse Mental Health Among More-Acculturated and Younger Immigrants Experiencing Discrimination: California Health Interview Survey, 2015-2016
}

\author{
Altaf Saadi, MD, MS ${ }^{\top}$ a and Ninez A. Ponce, MPP, PhD 2.3 \\ 'Department of Neurology , Massachusetts General Hospital, Harvard Medical School, Boston, MA, USA; ${ }^{2}$ UCLA Center for Health Policy Research, \\ Los Angeles, CA, USA; ${ }^{3}$ UCLA Fielding School of Public Health, Los Angeles, CA, USA.
}

BACKGROUND: Experiences of discrimination harm mental and physical health, with the strongest penalty on mental health. Among immigrants, it remains unclear how acculturation - the process by which immigrants acquire the beliefs and practices of a host culture-influences the mental health burden of navigating discrimination. On the one hand, acculturation can be associated with upward social mobility. Conversely, the acculturative process may increase exposure to, and recognition of, discrimination.

OBJECTIVES: We examined the relationship between discrimination and mental illness across racial/ethnic groups, and pathways by which acculturation and age relate to the discrimination-mental health relationship.

DESIGN: A secondary data analysis using population data from the 2015-2016 California Health Interview Survey.

MAIN MEASURES: The Kessler 6-item Psychological Distress Scale (K6) assessed symptoms of psychological distress, with $\mathrm{K} 6$ score $\geq 13$ associated with severe mental illness. Discrimination was measured using a self-reported measure of lifetime experience of unfair treatment in getting medical care. We used a 5-point acculturation index (constructed by measures of nativity, years living in the USA, and home language use). A weighted logistic regression model predicted mental illness as a function of discrimination. We ran mediational analysis using the Karlson-Holm-Breen method and used predictive margins to present predicted probabilities of mental illness for people reporting discrimination at different acculturation and age levels.

KEY RESULTS: There were independent effects on mental illness associated with increased discrimination (OR $3.85,95 \% \mathrm{CI}=2.46,6.03, p<0.001$ ) and increased acculturation (OR 1.72, 95\% CI $=1.24,2.38, p=0.001$ ), including when stratified across racial/ethnic groups. Higher levels of acculturation led to a significant increase in discrimination's association with mental illness. There was a higher probability of mental illness in younger age groups than in older age groups.

CONCLUSIONS: While discrimination is associated with poor mental health, a stronger link between discrimination and mental illness exists among younger immigrants and immigrants with increased acculturation. Health practitioners should not overlook the mental health needs

Electronic supplementary material The online version of this article (https://doi.org/10.1007/s11606-019-05412-w) contains supplementary material, which is available to authorized users.

Received May 27, 2019

Accepted September 20, 2019

Published online November 1, 2019 of younger immigrants and immigrants who may seem more integrated into US society.

KEY WORDS: discrimination; acculturation; immigrants; mental health.

J Gen Intern Med 35(5): 1419-26

DOI: $10.1007 / \mathrm{s} 11606-019-05412-w$

(C) Society of General Internal Medicine 2019

\section{INTRODUCTION}

Experiences of discrimination are an important type of psychosocial stressor that has adverse effects on both mental and physical health ${ }^{1}$. Systematic reviews reveal the strongest associations for harmful health effects are across a range of mental health outcomes including psychological distress, mental illness such as depression and anxiety, and suicidal ideation $^{1,2}$, with poor mental health as a consequence of discrimination seen among Black ${ }^{3}, 4$, Latino ${ }^{5,} 6$, Asian/ Pacific Islander ${ }^{7,8}$, American Indian ${ }^{9}$, Middle Eastern/Arab ${ }^{10}$, and White communities ${ }^{11}$. Factors influencing mental health impacts include coping style, sex, socioeconomic class, educational attainment, and racial/ethnic group affiliation ${ }^{1,5,12}$. One potential mechanism underlying the perceived discrimination-health relationship is allostatic load developed by heighted stress responses and negative emotional states from experiences of discrimination. Consequently, studies have suggested a dose-dependent relationship, with higher impacts of discrimination among older minority adults and/ or cumulative reported experiences of discrimination ${ }^{13,14}$.

Another contextual difference between racial/ethnic groups that may influence the experience of discrimination is immigration status and acculturation. Acculturation refers to the process by which immigrants acquire the language, customs, behaviors, and attitudes of the host culture consequent to contact with a host culture involving culturally dissimilar people and social influences ${ }^{15}$. There are both positive and negative theories of acculturation and how acculturation influences health. Positive theories include potential for upward social mobility, increased socioeconomic status or employment opportunities, increased social cohesion and community, increased understanding of the American healthcare system and preventive health practices, and development of an 
“American" identity ${ }^{16}$. Negative theories include adopting negative American behaviors such as change in diet or decreased physical activity, and increasing psychosocial stressors associated with acculturation ("acculturative stress") such as a change in self-worth or self-image due to changing roles from native to host country, the pressure of acquiring a new language, balancing differing cultural values, and increased exposure to, and recognition of, discrimination ${ }^{17-19}$. However, the pathways by which discrimination and acculturation affect mental health are not clear ${ }^{18}$, including whether or not there exists a dose-dependent relationship with older adults being disproportionately impacted.

The present study therefore aims to examine the pathways by which discrimination and acculturation affect mental health. We examine (1) the relationship between selfperceived discrimination and mental illness across racial/ ethnic groups, (2) whether acculturation mediates the effect of discrimination and mental illness, and (3) whether the perceived discrimination and mental illness relationship differ for different levels of acculturation and age.

\section{METHODS}

\section{Study Sample}

This study is a secondary analysis of a publicly available dataset, the 2015-2016 adult California Health Interview Survey (CHIS), which is the largest state health survey in the USA and the most recent complete 2-year cycle of the CHIS. The CHIS is a crosssectional, population-based telephone survey administered biannually and is approved by the Institutional Review Board of the University of California, Los Angeles (UCLA). It is conducted in six languages: English, Spanish, Chinese (Mandarin and Cantonese dialects), Vietnamese, Korean, and Tagalog. Participants in the CHIS are chosen at random. The adult response rate after screening the household was $47.2 \%$ in 2015 and $44.6 \%$ in $2016^{20}$. The sample includes both households with landlines and those with cell phones only. It collects information for all age groups on health status, health conditions, health-related behaviors, health insurance coverage, access to healthcare services, and other health-related issues for California's noninstitutionalized population. Our study subsample included only the 42,089 adults (ages 18-85) living in households in California who completed the 2015-2016 CHIS. A total of 274 adults were excluded from our study due to nonresponses for one of our main dependent variables (self-perceived discrimination).

\section{Measures}

The Kessler 6-item Psychological Distress Scale (K6) measured symptoms of psychological distress. The K6 measures psychological distress with six questions (e.g., "How often did you feel nervous?" "How often did you feel hopeless?"), scored on a 5point Likert scale ranging from none of the time to all of the time $^{21}$. Items are summed to obtain a total score ranging from 0 to 24 with each question scored from 0 ("none of the time") to 4 ("all of the item"). The $\mathrm{K} 6$ scale is a widely used indicator of nonspecific psychological distress, highly correlated with mental illness, and allows ranking of respondents on a distress scale continuum for research purposes ${ }^{21}$. Since a score of 13 or greater on the K6 has been shown to be a strong indicator of the presence of a diagnosable mental illness with considerable disability $^{22}$, we created a categorical variable $(\mathrm{K} 6 \geq 13, \mathrm{~K} 6<13)$.

Self-perceived discrimination was measured using one question in the CHIS 2015-2016 dataset- "How often were you treated unfairly when getting medical care over your lifetime?" Response categories ranged from 1 for "never" to 4 for "often." A follow-up question, asking the main reason for being treated unfairly, was used for descriptive statistics to contextualize the discrimination measure further, such as how often attributes associated with racial discrimination were cited (person's race/skin color, ancestry/national origin, and/ or English speaking ability). Multiple forms of discrimination, often overlapping among racially diverse samples, have been linked to poor mental health outcomes ${ }^{8,11}$.

The level of acculturation was assessed using three measures: place of birth (US born vs foreign born), duration of US residency (5 years, 5-9 years, and 10 or more years), and language spoken at home (speaks English only, speaks English and another language, and speaks a non-English language only), adapted from Erinosho et al. ${ }^{23}$ The duration of US residency cutoffs is based on the fact that it typically takes a minimum of 5 years to obtain US citizenship and research shows that after 10 years, the health of foreign-born immigrants tend to become similar to the US-born. A citizenship variable was not included directly into the index since this is more of a policy measure, with immigrants acculturating whether or not they obtain citizenship. Previous studies assessing optimal ways to measure acculturation have also indicated that multiple indicators of acculturation are useful, particularly when measures involve both language and duration/length of stay measures ${ }^{17}$.

Adults were assigned a score of $0-3$ based on their place of birth and duration of US residency: $0=$ foreign born, resided in the USA for $<5$ years; $1=$ foreign born, resided in the USA for $5-9$ years; 2 = foreign born, resided in the USA for $\geq 10$ years; and $3=$ US born. Language at home was assigned scores of 0 2: $0=$ speaks a non-English language only, $1=$ speaks English and another language, and $2=$ speaks English only. Scores assigned were assumed to create an acculturation score ranging from 0 (least acculturated) to 5 (most acculturation). Given skew towards more-acculturated individuals in the sample, the acculturation variable was dichotomized $(0=$ less acculturated, acculturation index score $0-3 ; 1=$ more acculturated, acculturation index score 4-5).

\section{Sociodemographic Control Variables}

We accounted for age (continuous), sex (male/female), education level (no high school diploma/high school graduate/some 
college/college and above), employment status (yes/no), insurance status (yes/no), federal poverty level (0-99\% of FPL/ 100-199\% FPL/200-299\% FPL/300\% FPL and above), and race/ethnicity. We used the California Department of Finance race/ethnicity classification, which tabulates Latino/Hispanic as a mutually exclusive racial/ethnic category from the nonLatino/Hispanic major race categories specified by the federal Office of Management and Budget. We decided to keep White adults in the model because experiences of discrimination affect both White individuals and Middle Eastern/Arab individuals who are classified as White by the US Census and subject to discrimination $^{11,24}$.

\section{Statistical Analysis}

Analyses began with descriptive statistics and bivariate associations between study measures. To assess the relationship between self-perceived discrimination (K6 $<13$ vs K6 $\geq 13$ ) and mental illness and acculturation across racial/ethnic groups, we used logistic regression and adjusted for covariates. We ran the same model stratified by racial/ethnic group to evaluate subgroup differences. To assess whether acculturation mediates the effect of discrimination and mental illness, we ran mediational analysis using the Karlson-Holm-Breen method $^{25}$, which decomposes the total effect of a variable into direct and indirect effects for a logistic regression model. Lastly, to best communicate and quantify whether the perceived discrimination and mental illness relationship differs for different levels of acculturation, we estimated marginal effects to express how the predicted probability of the outcome changes as acculturation level changes and across different age categories $^{26}$. Survey-supplied weights were used to produce population estimates ${ }^{27}$. All analyses were conducted by using Stata version 15.0 (StataCorp LP, College Station, TX).

\section{RESULTS}

Table 1 displays characteristics of the study sample, stratified by race/ethnicity. Whites were significantly older than the other racial/ethnic subgroups ( $p$ value $<0.001$, weighted sample mean 44.00, SE 0.032). White and Asian subgroups were the most college educated, with the Hispanic population having the highest percentage of individuals without a high school diploma. The sample was predominantly insured $(>85 \%$ across all groups), with Whites most likely to be insured and the largest difference with the Hispanic population. Similarly, there was significant stratification by poverty and race, with White income levels highest and the largest difference seen with the Hispanic population. White and Black populations had no significant difference in acculturation index scores (mostly acculturated, with $>85 \%$ US born and speaking English only at home) when compared with Hispanic, Asian, and other racial/ethnic populations. The largest acculturation gap was noted between Whites and Hispanics.
Among the total sample, $30.33 \%$ reported discrimination. Average reports of discrimination varied by race/ethnicity, with reports of discrimination lowest in the Asian group and highest in the Black and other racial/ethnic subgroups $(p<$ 0.001 , respectively). Attributes associated with racial/ethnic discrimination (person's race/skin color, ancestry/national origin, and/or English speaking ability) made up the plurality of responses at $30 \%$ of the domains for self-perceived discrimination. Among Whites, dissatisfaction with the healthcare system, gender/sex, and age were the three most common reasons cited for being discriminated against in the healthcare context.

In terms of psychological distress, the sample reported, on average, low K6 scale scores with a weighted mean K6 scale score of $4.44(\mathrm{SE}=0.056)$. Weighted mean scores were lower for Asians $(4.08, \mathrm{SE}=0.19 ; p$ value $<0.0001)$.

Table 2 shows both the adjusted and unadjusted models. Supplementary Tables 3, 4, 5, 6, 7, and 8 show our sensitivity analyses examining our model with the three measures of the acculturation index independently, and across racial/ethnic groups. In the unadjusted and adjusted logistic regression models, increased discrimination and acculturation were independently associated with increased odds of mental illness (K6 $\leq 13)(p<0.001$ and $p=0.001$ in adjusted model, respectively). Increased exposure to discrimination illustrates a gradient in the discrimination-mental illness association, such that those who reported higher frequencies of discrimination conferred increased odds of having mental illness. For example, reporting being treated unfairly "rarely" conferred a 1.91 increased odds of having mental illness whereas reporting unfair treatment "often" conferred a 3.85 increased odds of having mental illness, relative to those "never" reporting unfair treatment in the healthcare context (Table 2). Higher acculturation led to a 1.72 increased odds in mental illness relative to those with less acculturation ( $p$ value $=0.001$ ). The same relationship was observed when stratified across each racial/ethnic group. A goodness of fit test indicated that our model with its set of covariates fits the data well (Prob $>F=$ 0.71). There was no interaction between acculturation and racial/ethnic group or between discrimination and racial/ ethnic group (data not shown). Mediational analysis did not show evidence of acculturation mediating self-perceived discrimination's effect on mental illness (Fig. 1).

Different levels of acculturation led to significant change in rates of mental illness among those reporting self-perceived discrimination (Fig. 2). For example, among those reporting discrimination exposure "sometimes," higher acculturation predicted a $19.5 \%$ rate of mental illness relative to $9.5 \%$ for those less acculturated ( $p$ value $<0.0001$ ). Among those reporting discrimination exposure "frequently," higher acculturation predicted a $20.0 \%$ rate of mental illness relative to $17.2 \%$ for those less acculturated ( $p$ value $<0.0001$ ). Across four different age categories (age 18-39, 40-54, 55-69, and $70-85$ ), there was a higher probability of mental illness in younger age groups than in older age groups, including among 
Table 1 Population Characteristics of California Adults

\begin{tabular}{|c|c|c|c|c|c|c|}
\hline & $\begin{array}{l}\text { Non-Hispanic } \\
\text { White }\end{array}$ & Hispanic & $\begin{array}{l}\text { Non-Hispanic } \\
\text { Black }\end{array}$ & Asian & $\begin{array}{l}\text { Other } \\
\text { race/ethnicities }\end{array}$ & Total \\
\hline \multicolumn{7}{|l|}{ Demographic characteristics } \\
\hline Age, mean (SE) & $48.97(0.25)$ & $39.59(0.27)$ & $44.50(0.87)$ & $40.83(0.54)$ & 40.95 (1.09) & $44.00(0.032)$ \\
\hline Male, $\%$ & 49.26 & 49.71 & 46.81 & 46.28 & 47.66 & 48.81 \\
\hline \multicolumn{7}{|l|}{ Education level, \% } \\
\hline Less than HS & 4.53 & 37.66 & 11.43 & 7.30 & 7.11 & 17.12 \\
\hline HS graduate & 19.94 & 26.06 & 25.52 & 16.09 & 20.70 & 21.89 \\
\hline Some college & 26.69 & 19.98 & 32.60 & 17.77 & 35.06 & 23.62 \\
\hline College graduate or higher & 48.84 & 16.29 & 30.45 & 58.84 & 37.13 & 37.36 \\
\hline Employed, \% & 61.32 & 68.56 & 61.96 & 67.35 & 63.43 & 64.84 \\
\hline Insured, \% & 95.14 & 82.71 & 93.38 & 93.47 & 92.52 & 90.33 \\
\hline \multicolumn{7}{|l|}{ Federal poverty level, $\%$} \\
\hline $0-99 \% \mathrm{FPL}$ & 8.81 & 28.61 & 24.11 & 14.58 & 18.08 & 17.77 \\
\hline $100-199 \% \mathrm{FPL}$ & 11.40 & 28.80 & 11.40 & 16.24 & 18.99 & 18.90 \\
\hline $200-299 \%$ FPL & 12.14 & 16.13 & 12.14 & 11.52 & 13.53 & 13.67 \\
\hline$\geq 300 \% \mathrm{FPL}$ & 67.65 & 26.46 & 67.65 & 57.66 & 49.41 & 49.66 \\
\hline \multicolumn{7}{|l|}{ Acculturation index, \% } \\
\hline Least acculturated $(0)$ & 0.48 & 1.49 & 0.042 & 3.49 & 0.00 & 1.23 \\
\hline 1 & 0.57 & 3.23 & 0.38 & 7.54 & 0.094 & 2.48 \\
\hline 2 & 1.59 & 24.06 & 1.78 & 21.88 & 0.59 & 12.42 \\
\hline 3 & 3.85 & 28.60 & 2.6 & 29.03 & 4.09 & 16.14 \\
\hline 4 & 10.18 & 25.21 & 8.13 & 21.54 & 15.56 & 17.16 \\
\hline Most acculturated (5) & 83.33 & 17.40 & 87.07 & 16.51 & 79.67 & 50.57 \\
\hline \multicolumn{7}{|c|}{ Self-reported experiences of discrimination, $\%$} \\
\hline Never & 71.04 & 68.95 & 59.89 & 78.27 & 59.72 & 70.38 \\
\hline Rarely & 19.05 & 17.65 & 24.22 & 13.13 & 22.79 & 18.11 \\
\hline Sometimes & 8.08 & 10.70 & 12.51 & 7.44 & 13.10 & 9.31 \\
\hline Often & 1.83 & 2.69 & 3.37 & 1.16 & 4.39 & 2.20 \\
\hline \multicolumn{7}{|c|}{ Kessler 6-item Psychological Distress Scale score } \\
\hline Mean (SE) & $4.31(0.088)$ & $4.54(0.092)$ & $5.07(0.26)$ & $4.08(0.19)$ & $5.59(0.35)$ & $4.44(0.056)$ \\
\hline$<13$ & 91.57 & 91.72 & 88.60 & 93.54 & 88.88 & 91.66 \\
\hline$\geq 13$ & 8.43 & 8.28 & 11.40 & 6.46 & 11.12 & 8.34 \\
\hline
\end{tabular}

Source: 2015-2016 California Health Interview Survey; weighted estimates

those with higher acculturation (Fig. 2). That is, among those reporting discrimination exposure "sometimes," being less acculturated and in the 18-39 age category conferred a risk of mental illness at $13 \%$ relative to those who were more acculturated and in the same age category who had a predicted risk of mental illness of $26.5 \%$ ( $p$ value $<0.0001$ ). Conversely, among those reporting the same discrimination exposure ("sometimes") but in the 70-85 age category, the predicted risk of mental illness for those who were less acculturated was $4.6 \%$ and, for those who were more acculturated, was $10.6 \%$ ( $p$ value $<0.0001)$

\section{DISCUSSION}

Our analysis yielded three major findings: (1) more frequent self-perceived discrimination was associated with increased odds of mental illness, across racial/ethnic groups; (2) acculturation did not mediate the effect of discrimination and mental illness; and (3) the perceived discrimination and mental illness relationship was more profound among those with higher acculturation and younger age.

Our results bolster previous studies supporting the relationship between discrimination and mental health ${ }^{1,2}$, including that increased exposure to discrimination results in poorer mental health outcomes. This study adds to the literature by including analysis of multiple ethnic minority groups, including White and other racial/ethnic subgroups often excluded due to a focus on sizeable minority communities in the USA. This focus comes at the expense of Middle Eastern populations, categorized as White in the US Census, who experience discrimination and consequent negative mental health outcomes ${ }^{10}$, as well as mixed-race individuals also experiencing discrimination ${ }^{28}$. Both racial/ethnic and non-racial/ethnic discrimination can be associated with worse mental health ${ }^{14}$. Further, even White individuals themselves experience discrimination and can have poor mental health as a result - these discriminatory experiences can relate to other aspects of one's identity such as income, weight, or gender. Future studies elucidating the differential impact of different domains of discrimination experience, beyond racial/ethnic discrimination, would be informative.

The findings of this study build upon research that has examined the relationship of discrimination to negative mental health outcomes as influenced by acculturation. Specifically, we did not observe a mediating relationship between acculturation and discrimination's impact on mental illness, rather suggesting that higher levels of acculturation play a deleterious effect on discrimination's impact on mental health. This complements some prior studies showing that moreacculturated Mexican immigrants reported higher rates of discrimination that less-acculturated immigrants ${ }^{29}$. Reasons for this include increased assimilation in US society resulting in increased perception of American racial dynamics, 
Table 2 The Association of Discrimination and Acculturation on Mental Illness (K6 $\geq 13$ ) Among California Adults

\begin{tabular}{|c|c|c|c|c|c|c|}
\hline \multirow[t]{2}{*}{ Variable } & \multicolumn{2}{|c|}{$\begin{array}{l}\text { Model 1, unadjusted, } \\
\text { discrimination only }\end{array}$} & \multicolumn{2}{|c|}{$\begin{array}{l}\text { Model 2, unadjusted, } \\
\text { discrimination and } \\
\text { acculturation only }\end{array}$} & \multicolumn{2}{|c|}{$\begin{array}{l}\text { Model 3, adjusted, discrimination, } \\
\text { acculturation, and covariates }\end{array}$} \\
\hline & OR (SE) & $95 \%$ CI ( $p$ value) & OR (SE) & $95 \%$ CI ( $p$ value) & OR (SE) & $95 \%$ CI ( $p$ value) \\
\hline \multicolumn{7}{|l|}{$\begin{array}{l}\text { Self-perceived discrimination } \\
\text { (reference = never) }\end{array}$} \\
\hline Rarely & $1.97(0.27)$ & $1.50,2.58(<0.001)$ & $1.94(0.26)$ & $1.47,2.54(<0.001)$ & $1.91(0.27)$ & $1.44,2.52(<0.001)$ \\
\hline Sometimes & $3.40(0.40)$ & $2.68,4.31(<0.001)$ & $3.51(0.41)$ & $2.78,4.44(<0.001)$ & $3.08(0.39)$ & $2.40,3.96(<0.001)$ \\
\hline Often & $4.97(1.05)$ & $3.27,7.56(<0.001)$ & $5.04(1.03)$ & $3.35,7.59(<0.001)$ & $3.85(0.87)$ & $2.46,6.03(<0.001)$ \\
\hline \multicolumn{7}{|l|}{$\begin{array}{l}\text { Acculturation (reference = } \\
\text { less acculturated) }\end{array}$} \\
\hline $\begin{array}{l}\text { More acculturated } \\
\quad \text { (acculturation index 4-5) }\end{array}$ & & & $1.64(0.17)$ & $1.33,2.02(<0.001)$ & $1.72(0.28)$ & $1.24,2.38(0.001)$ \\
\hline \multicolumn{7}{|l|}{ Covariates } \\
\hline \multicolumn{7}{|l|}{$\begin{array}{l}\text { Race/ethnicity (reference } \\
\text { = White) }\end{array}$} \\
\hline Hispanic & & & & & $0.69(0.10)$ & $0.52,0.91(<0.01)$ \\
\hline Black & & & & & $0.90(0.17)$ & $0.61,1.32(0.59)$ \\
\hline Asian & & & & & $0.83(0.20)$ & $0.51,1.34(0.44)$ \\
\hline Other & & & & & $0.84(0.21)$ & $0.51,1.40(0.51)$ \\
\hline Age & & & & & $0.97(0.002)$ & $0.96,0.98(<0.001)$ \\
\hline Sex $($ reference $=$ male $)$ & & & & & $1.16(0.12)$ & $0.94,1.44(0.15)$ \\
\hline \multicolumn{7}{|c|}{$\begin{array}{l}\text { Educational attainment } \\
\text { (reference = college and above) }\end{array}$} \\
\hline No high school diploma & & & & & $1.76(0.33)$ & $1.21,2.55(<0.005)$ \\
\hline HS graduate & & & & & $1.29(0.17)$ & $0.99,1.68(0.056)$ \\
\hline Some college & & & & & $1.44(0.24)$ & $1.04,2.02(<0.05)$ \\
\hline Poverty level & & & & & $0.80(0.03)$ & $0.74,0.87(<0.001)$ \\
\hline $\begin{array}{l}\text { Employment (reference } \\
=\text { employed) }\end{array}$ & & & & & $1.56(0.19)$ & $1.23,1.98(<0.001)$ \\
\hline $\begin{array}{l}\text { Insurance (reference } \\
=\text { insurance) }\end{array}$ & & & & & $0.83(0.14)$ & $0.60,1.16(0.28)$ \\
\hline
\end{tabular}

Source: California Health Interview Survey 2015-2016; weighted estimates

alongside emergence of a racial identity and increased perception of different or unfair treatment. In one study focusing on Latino and Black individuals, the association of healthcare discrimination with poorer mental health was weaker for recent immigrants ${ }^{6}$. The authors suggested that new immigrants might be able to protect against the mental health effects

Total effect of Self-Perceived Discrimination on Mental Illness

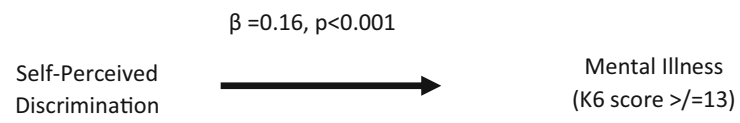

Direct and indirect effects of Total effect of Self-Perceived Discrimination on Mental Illness, mediated by Acculturation

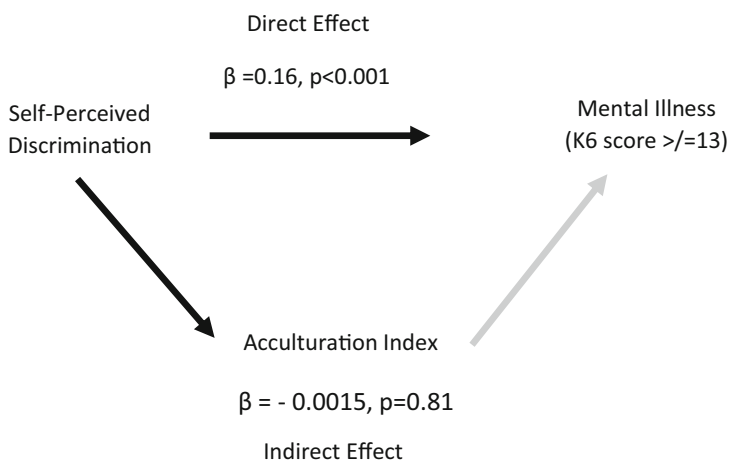

*Adjusted for age, sex, race/ ethnicity, education, employment,poverty level, and insurance status

Figure 1 Mediational analysis of effect of self-perceived discrimination and mental illness, California adults. Source: California Health Interview Survey 2015-2016. Adjusted for age, sex, race/ethnicity, education, employment, poverty level, and insurance status 

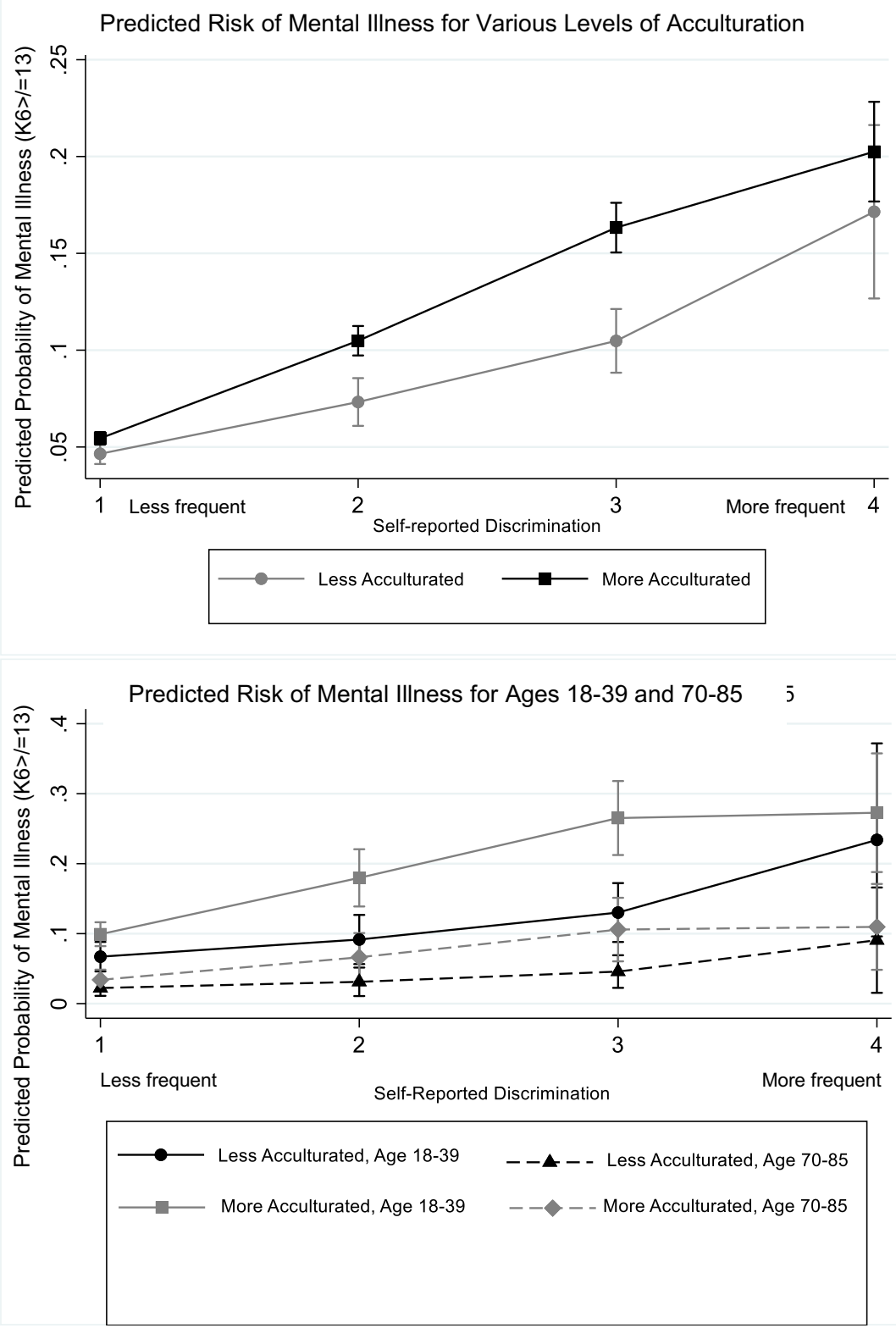

Figure 2 Predicted risk of mental illness across age and acculturation levels. Source: California Health Interview Survey 2015 -2016.

of discrimination by perceiving their negative experiences as stemming from unfamiliarity with US culture rather than their race/ethnicity or other personal identity factors.

Increased acculturation may also lead to more intermingling with individuals from other racial/ethnic groups, and thus increased exposure to interactions that may be discriminatory, or development of a racial identity better attuned to complex racial dynamics in the US ${ }^{16}$. In fact, these increased exposures may come even as someone gains social mobility on the one hand, via improved English proficiency, educational attainment, and/ or employment opportunities; on the other hand, increasing social contacts may increase the odds of encountering discrimination and subsequent negative health consequences.

Increased social mobility may also lead to comparisons with reference groups (i.e., individuals with similar level of English proficiency and/or higher levels of education either in their home countries or in the US), called the relative deprivation theory ${ }^{30}$. Other studies point to shifting and eroding family bonds with greater time in the US, diminishing the protective effect of familial social networks ${ }^{31}$. There have also been studies revealing acculturative stress associated with maintaining dual or multiple identities and/or languages ${ }^{32}$.

The relationship between acculturation and discrimination's impact on mental health may also not be unidirectional. That is, perceived discrimination may influence one's level of acculturative stress just as acculturative stress heightens impact of discrimination on mental health. We understand these as overlapping but distinct etiologies with impacts on mental health, which further studies can continue to clarify.

Lastly, our study found that the negative impact of higher acculturation on the mental health consequences of discrimination is concentrated among younger adults rather than older 
adults, which suggests that this is not just an exposure issue as identified in previous studies ${ }^{14}$. This penalty on younger adults may be explained by different coping skills between younger and older adults ${ }^{33}$, differences in social networks between those age groups ${ }^{34}$, and the experience of discrimination being more proximal or salient for younger adults, thereby increasing its impact on their mental health. There is also some evidence to suggest that younger, more-acculturated individuals could experience higher psychological distress in family situations where there is an acculturation gap or acculturation dissonance between the parent (less acculturated) and the young adult child (more acculturated) ${ }^{35}$.

There are limitations to our study. First, causal conclusions cannot be determined given the cross-sectional design that does not examine temporal effects. Second, we do not explore withingroup heterogeneity, which may influence experiences of discrimination and acculturation, such as among different Hispanic or Asian groups ${ }^{8}$. Third, our proxy measure for acculturation assumes a linear process, when the process of acculturation is more complex and multidimensional. For example, segmented assimilation posits a path of acculturation characterized by rapid economic and educational advancement while intentionally maintaining ethnic values ${ }^{16}$. Biculturalism suggests that individuals can be oriented toward both their cultures of origin and host culture simultaneously ${ }^{17}$. These individuals who have high levels of orientation to both mainstream US and traditional cultures may experience decreased acculturative stress and healthier outcomes because of their fluency in both cultures ${ }^{18}$. A more comprehensive evaluation of social networks and/or cultural values may outline specific cultural features that are associated with mental health, and how mainstream versus original culture orientation differently impacts perceived discrimination. Fourth, using a self-reported, single-item discrimination measure may introduce response and measurement biases, not completely capturing the full range of discrimination experiences $^{36,37}$. Further, severe events are better recalled than less severe ones, so a single-item measure may not accurately capture the effect of everyday discriminatory incidents that may have a differential impact on mental health than major lifetime discrimination $^{37}$. This is not even considering multiple levels of discrimination that operate simultaneously, such as institutional discrimination that should be acknowledged alongside our study focus on self-reported, individual-level discrimination ${ }^{8}$.

With these caveats in mind, our findings from a large, representative dataset have important public health implications. The current findings suggest that the mental health needs of immigrants who may seem more integrated into US society should not be overlooked and that individuals need not have a long, lifetime experience to discrimination for it to negatively impact their mental health.

Corresponding Author: Altaf Saadi, MD, MS; Department of Neurology Massachusetts General Hospital, Harvard Medical School, 55 Fruit St, WACC \#720, Boston, MA 02120, USA (e-mail: asaadi@mgh.harvard.edu).

\section{Compliance with Ethical Standards:}

Conflict of Interest: The authors declare that they do not have a conflict of interest.

\section{REFERENCES}

1. Pascoe EA, Richman LS. Perceived Discrimination and Health: A MetaAnalytic Review. Psychol Bull. 2009;135(4):531-554. doi:https://doi.org/ 10.1037/a0016059

2. Williams DR, Neighbors HW, Jackson JS. Racial/ethnic discrimination and health: findings from community studies. Am J Public Health. 2003;93(2):200-208

3. Banks KH, Kohn-Wood LP, Spencer M. An examination of the African American experience of everyday discrimination and symptoms of psychological distress. Community Ment Health J. 2006;42(6):555-570. doi:https://doi.org/10.1007/s10597-006-9052-9

4. Williams DR, Williams-Morris R. Racism and mental health: the African American experience. Ethn Health. 2000;5(3-4):243-268. doi:https://doi. org/10.1080/713667453

5. Ward JB, Feinstein L, Vines AI, Robinson WR, Haan MN, Aiello AE. Perceived discrimination and depressive symptoms among US Latinos: the modifying role of educational attainment. Ethn Health. 2019;24(3):271-286. doi:https://doi.org/10.1080/13557858.2017. 1315378

6. Gee GC, Ryan A, Laflamme DJ, Holt J. Self-Reported Discrimination and Mental Health Status Among African Descendants, Mexican Americans, and Other Latinos in the New Hampshire REACH 2010 Initiative: The Added Dimension of Immigration. Am $J$ Public Health. 2006;96(10):1821-1828. doi:https://doi.org/10.2105/AJPH.2005. 080085

7. Gee GC, Spencer MS, Chen J, Takeuchi D. A Nationwide Study of Discrimination and Chronic Health Conditions Among Asian Americans. Am J Public Health. 2007;97(7):1275-1282. doi:https://doi.org/10. 2105/AJPH.2006.091827

8. Gee GC, Ro A, Shariff-Marco S, Chae D. Racial Discrimination and Health Among Asian Americans: Evidence, Assessment, and Directions for Future Research. Epidemiol Rev. 2009;31:130-151. doi:https://doi. org/10.1093/epirev/mxp009

9. Johansson $\mathbf{P}$, Muller CJ, Samos MW, Goldberg $\mathbf{J}$. The influence of perceived discrimination on health-related quality of life in an East Coast American Indian tribe. J Health Care Poor Underserved. 2013;24(4):15311541. doi:https://doi.org/10.1353/hpu.2013.0183

10. Samari G, Alcalá HE, Sharif MZ. Islamophobia, Health, and Public Health: A Systematic Literature Review. Am $J$ Public Health. 2018;108(6):e1-e9. doi:https://doi.org/10.2105/AJPH.2018.304402

11. Bower KM, Thorpe RJ, LaVeist TA. Perceived Racial Discrimination and Mental Health in Low-income Urban-dwelling Whites. Int $J$ Health Serv Plan Adm Eval. 2013;43(2):267-280.

12. Noh S, Kaspar V. Perceived Discrimination and Depression: Moderating Effects of Coping, Acculturation, and Ethnic Support. Am J Public Health 2003;93(2):232-238.

13. Beatty Moody DL, Waldstein SR, Tobin JN, Cassells A, Schwartz JC, Brondolo E. Lifetime racial/ethnic discrimination and ambulatory blood pressure: The moderating effect of age. Health Psychol Off $J$ Div Health Psychol Am Psychol Assoc. 2016;35(4):333-342. doi:https://doi.org/10. $1037 /$ hea0000270

14. Wallace S, Nazroo J, Bécares L. Cumulative Effect of Racial Discrimination on the Mental Health of Ethnic Minorities in the United Kingdom. Am J Public Health. 2016;106(7):1294-1300. doi:https://doi.org/10. 2105/AJPH.2016.303121

15. Schwartz SJ, Unger JB, Zamboanga BL, Szapocznik J. Rethinking the Concept of Acculturation. Am Psychol. 2010;65(4):237-251. doi:https:// doi.org/10.1037/a0019330

16. Abraído-Lanza AF, Echeverría SE, Flórez KR. Latino Immigrants, Acculturation, and Health: Promising New Directions in Research. Annu Rev Public Health. 2016;37:219-236. doi:https://doi.org/10.1146/ annurev-publhealth-032315-021545

17. Lee S, O'Neill AH, Ihara ES, Chae DH. Change in Self-Reported Health Status among Immigrants in the United States: Associations with Measures of Acculturation. PLOS ONE. 2013;8(10). doi:https://doi.org/ 10.1371/journal.pone.0076494 
18. Torres L, Driscoll MW, Voell M. Discrimination, Acculturation, Acculturative Stress, and Latino Psychological Distress: A Moderated Mediational Model. Cultur Divers Ethnic Minor Psychol. 2012;18(1):17-25. doi:https://doi.org/10.1037/a0026710

19. Gee GC, Ponce N. Associations Between Racial Discrimination, Limited English Proficiency, and Health-Related Quality of Life Among 6 Asian Ethnic Groups in California. Am J Public Health. 2010;100(5):888-895. doi:https://doi.org/10.2105/AJPH.2009.178012

20. California Health Interview Survey. CHIS 2015-2016 Methodology Series: Report 4 -Response Rates. 2017. http://healthpolicy.ucla.edu/chis/ design/Documents /CHIS_2015-2016_MethodologyReport4_ ResponseRates.pdf. .

21. Kessler RC, Andrews G, Colpe LJ, et al. Short screening scales to monitor population prevalences and trends in non-specific psychological distress. Psychol Med. 2002;32(6):959-976.

22. Pratt LA. Serious psychological distress, as measured by the K6, and mortality. Ann Epidemiol. 2009;19(3):202-209. doi:https://doi.org/10. 1016/j.annepidem.2008.12.005

23. Erinosho TO, Berrigan D, Thompson FE, Moser RP, Nebeling LC, Yaroch AL. Dietary Intakes of Preschool-Aged Children in Relation to Caregivers' Race/Ethnicity, Acculturation, and Demographic Characteristics: Results from the 2007 California Health Interview Survey. Matern Child Health J. 2012;16(9):1844-1853. doi:https://doi.org/10.1007/ s10995-011-0931-5

24. Byrd DR. Race/Ethnicity and Self-Reported Levels of Discrimination and Psychological Distress, California, 2005. Prev Chronic Dis. 2012;9. doi:https://doi.org/10.5888/pcd9.120042

25. Kohler U, Karlson $\mathbf{K}$. KHB: Stata module to decompose total effects into direct and indirect via KHB-method. February 2019.

26. Norton EC, Dowd BE, Maciejewski ML. Marginal Effects-Quantifying the Effect of Changes in Risk Factors in Logistic Regression Models. JAMA. March 2019. doi:https://doi.org/10.1001/jama.2019.1954

27. California Health Interview Survey. CHIS 2015-2016 Methodology Series: Report 5 -Weighting and Variance Estimation. 2017. http://healthpolicy. ucla.edu/chis/design/Documents/CHIS_2015-2016_ MethodologyReport5_WeightingAndVarianceEstimation.pdf. .

28. Veenstra G. Black, White, Black and White: mixed race and health in Canada. Ethn Health. 2019;24(2):113-124. doi:https://doi.org/10.1080/ 13557858.2017 .1315374
29. Finch BK, Kolody B, Vega WA. Perceived discrimination and depression among Mexican-origin adults in California. $J$ Health Soc Behav. 2000;41(3):295-313.

30. Zhang W, Hong S. Perceived Discrimination and Psychological Distress Among Asian Americans: Does Education Matter? J Immigr Minor Health. 2013;15(5):932-943. doi:https://doi.org/10.1007/s10903-012-9676-5

31. Bostean G, Gillespie BJ. Acculturation, acculturative stressors, and family relationships among Latina/o immigrants. Cultur Divers Ethnic Minor Psychol. 2018;24(1):126-138. doi:https://doi.org/10.1037/ cdp0000169

32. Tikhonov AA, Espinosa A, Huynh Q-L, Anglin DM. Bicultural identity harmony and American identity are associated with positive mental health in U.S. racial and ethnic minority immigrants. Cultur Divers Ethnic Minor Psychol. February 2019. https://doi.org/10.1037/cdp0000268

33. Birditt KS. Age Differences in Emotional Reactions to Daily Negative Social Encounters. J Gerontol B Psychol Sci Soc Sci. 2014;69(4):557-566. doi:https://doi.org/10.1093/geronb/gbt045

34. Fung HH, Carstensen LL, Lang FR. Age-Related Patterns in Social Networks among European Americans and African Americans: Implications for Socioemotional Selectivity across the Life Span. Int J Aging Hum Dev. 2001;52(3):185-206. doi:https://doi.org/10.2190/1ABL-9BE5MOX2-LR9V

35. Telzer EH. Expanding the Acculturation Gap-Distress Model: An Integrative Review of Research. Hum Dev. 2010;53(6):313-340. doi:https:// doi.org/10.1159/000322476

36. Bastos JL, Celeste RK, Faerstein E, Barros AJD. Racial discrimination and health: a systematic review of scales with a focus on their psychometric properties. Soc Sci Med 1982. 2010;70(7):1091-1099. https://doi.org/10.1016/j.socscimed.2009.12.020

37. Gee GC. A multilevel analysis of the relationship between institutional and individual racial discrimination and health status. Am $J$ Public Health. 2002;92(4):615-623.

Publisher's Note: Springer Nature remains neutral with regard to jurisdictional claims in published maps and institutional affiliations. 